\title{
Bildung, Beruf und Arbeitseinkommen: Theoretische Verknüpfungen zwischen Aspekten der sozialen Schichtung*
}

\author{
Volker Bornschier \\ Universität Zürich, Soziologisches Institut \\ Zeltweg 63, CH-8032 Zürich
}

$\mathrm{Z}$ u s a m m e $\mathrm{n}$ f a $s \mathrm{~s}$, $\mathrm{n}$ g: Der Aufsatz behandelt die Verknüpfung zwischen zwei wichtigen Dimensionen der sozialen Schichtung, der Bildung und dem Einkommen. Zwei für die Analyse der Sozialstruktur zentrale Fragen Was begründet den Zusammenhang zwischen formaler Bildung und dem Arbeitseinkommen? Warum ist dieser Zusammenhang nicht besonders stark? - werden anhand von vier Theorien diskutiert. Es handelt sich einmal um die Legitimationstheorie, weiter um die Humankapital-Theorie und um zwei Versionen einer Auswahltheorie. Die eine Auswahltheorie argumentiert von der organisationellen Arbeitsteilung her und die andere auf der Grundlage einer Arbeitskräftewarteschlange beim Wettbewerb um Arbeitsplätze. Die Theorien decken einen weiten Bereich möglicher Argumentationsfiguren bei soziologischen wie ökonomischen Betrachtungen des Problems ab. Die theoretischen Argumente, die einerseits in makro-, andererseits in mikrotheoretischen Vorstellungen gründen, unterscheiden sich teilweise beträchtlich in der Herleitung der Verknüpfung und mit Blick auf die Stärke der erwarteten Beziehung wie auch bei der Frage, welchen Einfluß eine Änderung der Bildungsverteilung für die Einkommensverteilung hat. Die Behandlung der Theorien verfolgt das Ziel einer teilweisen theoretischen Integration einerseits und einer klaren theoretischen Trennung andererseits, um Arbeitshypothesen für die empirische Forschung anzuregen, die helfen, die Kräfte besser zu ergründen, die der sozialen Schichtung zugrundeliegen.

\section{Einführung}

Bildung, Beruf und Arbeitseinkommen (wie auch das gesamte Einkommen) stehen im Mittelpunkt des Interesses bei der mehrdimensionalen Untersuchung der sozialen Schichtung wie auch bei den sogenannten Statuszuweisungsmodellen, die nach der Pionierarbeit von Blau und Duncan (1967) in der Soziologie populär wurden.

Dennoch fältt auf, daß die Theorie, die die Verknüpfungen zwischen diesen verschiedenen Dimensionen der Schichtung erklären soll, weniger ausgearbeitet ist als die vergleichsweise entwickelten empirischen Modelle. Das Kernargument, das die Verknüpfung begründet zwischen: sozialer Herkunft $\rightarrow$ erreichtem Schulabschluß $\rightarrow$ Beruf $\rightarrow$ Einkommen (Verdienst), ist sowohl in Arbeiten von Soziologen wie auch Ökonomen zu finden. Allerdings beobachtet man, daß Soziologen häufig ihre Modelle nur bis zum Beruf ausarbeiten und so die Einkommen und Verdienste nicht untersuchen, während die Ökonomen meistens den Beruf in ihren Modellen auslassen, also überspringen.

* Dies ist die deutsche Ubersetzung eines Referates, das auf der Konferenz des Research Committee on Social Statification der International Sociological Association in Paris im April 1981 gehalten wurde.
In diesem Aufsatz behandele ich zwei Fragen. Die erste kann folgendermaßen formuliert werden:

1. Welches sind die theoretischen Begründungen für soziale Verknüpfungen zwischen formaler Schulbildung und Einkommen, die sich empirisch in einer Korrelation zwischen beiden Aspekten der sozialen Schichtung ausdrücken? ${ }^{1}$

Es gibt viele Evidenzen für eine signifikante Korrelation zwischen formaler Schulbildung und der Höhe des Einkommens. Obwohl diese Korrelation, die man in verschiedenen Gesellschaften in neuerer Zeit ermittelt hat, im statistischen Sinne aussagekräftig ist, kann man

1 Im folgenden wird in der Regel nur die Verteilung der Arbeitseinkommen thematisiert, auch wenn nur die Kurzform „Einkommen" verwendet wird. Die gesamte Einkammensverteilung schließt neben den Arbeitseinkommen (zu denen konzeptuell auch Nebenvergünstigungen und andere geldwerte Belohnungen aus dem Arbeitszusammenhang zu zählen sind) die Kapitaleinkommen (einschließlich Bodenrente) und die Transfereinkommen (Zahlungen aus öffentlichen Sozialversicherungen und Subventionen) ein. Bei einer Lohnquote in den hochentwikkelten kapitalistischen Ländern von bis zu $80 \%$ des Gesamteinkommens kommt den Arbeitseinkommen im Rahmen der gesamten Einkommensverteilung eine überragende Bedeutung $\mathrm{zu}$. Unter formaler Bildung werden allgemeine wie auch berufsbezogene Ausbildungsgänge zusammengefaßt. 
dennoch nur einen vergleichsweise kleinen Teil der Einkommensunterschiede auf Unterschiede in der formalen Bildung zurückführen. Dieser Sachverhalt führt mich zur zweiten Frage:

2. Wenn die formale Bildung im Alltagsverständnis wie auch im Denken der soziologischen Profession eine solch bedeutsame Rolle beim Zugang zum Einkommen spielt, warum erklären dann Bildungsunterschiede nur einen so kleinen Teil der Einkommensunterschiede?

Sofort ist man versucht $\mathrm{zu}$ argumentieren, daß die formale Bildung nur mit Verdienstunterschieden in Zusammenhang steht und weniger Einkommensunterschiede erklärt, weil letztere mitbestimmt werden durch große Unterschiede bei der Vermögensverteilung. Dies erscheint als ein plausibler Einwurf. Unglücklicherweise hilft das Argument aber nicht bei der Lösung des Problems, das mit der zweiten Frage angeschnitten wird.

Zum Beispiel kommt Wilson (1978) bei seiner Untersuchung einer Alterskohorte von 32-jährigen Männem in den Vereinigten Staaten zum Ergebnis, daß zwischen erreichtem Bildungsabschluß und der Höhe des Jahreseinkommens nur eine gemeinsame Varianz von $18 \%$ besteht, und zwar sogar dann, wenn Bildung neu geeicht wird, um besser als z.B. mit der bloßen Zahl der Schuljahre oder mit dem höchsten erreichten Schulabschluß die Fähigkeit zum Einkommenserwerb zu repräsentieren. Zwar mißt Wilson (1978) das Einkommen und nicht den Verdienst, aber bei seiner vergleichsweise jungen Alterskohorte dürften die Einkommen noch kaum wesentlich durch Unterschiede beim Einkommen aus Vermögen beeinflußt sein. Eine solche Schlußfolgerung wird unterstützt durch die Ergebnisse von Griliches (1977). Er betrachtet nur die Verdienste und kommt für die Vereinigten Staaten zu einem ganz ähnlichen Ergebnis mit einer Stichprobe von 3025 Männern im Alter von 30 Jahren. Formale Bildung und Verdienste weisen eine gemeinsame Varianz von $18 \%$ auf $^{2}$. Man ist versucht zu sagen: von nur $18 \%$, wenn man bedenkt, welch große Rolle die Bildung für den Zugang zu gesellschaftlichen Belohnungen in

2 Diese geringe gemeinsame Varianz in einer Alterskohorte ist umso erstaunlicher, als Wilson (1978) und Griliches (1977) den Logarithmus des Einkommens bzw. der Verdienste verwenden. unserem Denken spielt ${ }^{3}$. Auch wenn man die Messung der Bildung ausweitet, nämlich zusätzlich zur formalen Schulbildung noch die Ausbildung am Arbeitsplatz einschließt (on-the-job training), auch dann ist Mincer $(1974,1976)$ in seinen Studien nur in der Lage, ein Drittel der Verdienstunterschiede auf eine so erweiterte Vorstellung von Bildung zurückzuführen (vgl. auch Sahota 1978: 13).

Im folgenden werde ich vier Theorien in den Grundzügen darstellen, um abzuklären, was sie zur Beantwortung der beiden vorher genannten Fragen beitragen können. Zwei dieser Theorien weisen im Hinblick auf die anstehenden Probleme eher einen makrotheoretischen Ansatz auf. Sie begründen, wie das Verhältnis von Bildung und Einkommen im sozialen Aggregat der Gesellschaft entsteht. Sie führen aber keine Uberlegungen zu vermittelnden Mechanismen ein, d.h. in diesen Modellen werden keine Aussagen. über Arbeitsplätze und Arbeitgeber gemacht. Die erste zu behandelnde Theorie ist die Sozialstruktur- und Legitimationstheorie, die von Peter Heintz entwickelt worden ist (Heintz, 1972a, 1972b, 1982; Bornschier und Heintz, 1977). Die zweite ist die Humankapital-Theorie, die von Gary Becker (1964), Jacob Mincer $(1974,1976)$ und ihren Schülern ausformuliert worden ist.

Die beiden übrigen Theorien sind - zumindest in den hier besonders in den Vordergrund gestellten Aspekten - mehr mikrotheoretisch. Sie

3 Die ausgewählten Beispiele untertreiben kaum die Höhe der gemeinsamen Varianz. Für die Bundesrepublik Deutschland kommt Müller (1975: 123) auf eine gemeinsame Varianz von gut $15 \%$ zwischen der formalen Bildung vor dem Berufseintritt und dem Einkommen einer repräsentativen Auswahl von Männern im Alter von 33 Jahren. Für Großbritannien ermittelt Psacharopoulos (1977) bei 25- bis 65-jährigen Männern eine gemeinsame Varianz von $16 \%$ zwischen formaler Bildung und Verdiensten. Für eine repräsentative Auswahl von männlichen $B e-$ schäftigten über 18 Jahre in den Vereinigten Staaten ermitteln Robinson und Kelley (1979: 47) nur eine gemeinsame Varianz von $13 \%$, vgl. auch die Studie von Lord und Falk (1980) mit noch niedrigeren Ergebnissen für die USA. Die Verhältnisse in Australien sind von Headey und O'Loughlin (1978) untersucht worden mit dem Resultat einer gemeinsamen Varianz in der Größenordnung zwischen 11-13\%. Dies ist keine abschließende Liste von Studien, die herangezogen werden können. 
berücksichtigen die Art und Weise, wie Entscheidungen auf dem Arbeitsmarkt beeinflußt werden und führen explizit die Arbeitgeber ein. So kommen sie auch zu Schlußfolgerungen, wie die Beziehung zwischen Bildung und Erwerbseinkommen im sozialen Aggregat entsteht. Die eine Theorie wird Arbeitsteilungs- und Auswahltheorie der Bildung genannt (Bornschier 1977, 1981), die andere: Arbeitsplatzwettbewerbs-, Arbeitskräftewarteschlangen- und Indikatortheorie der Bildung (Thurow 1975, 1978).

\section{Sozialstruktur- und Legitimationstheorie}

Die Sozialstruktur- und Legitimationstheorie betrachtet die Ausgliederung von sozioökonomischen Statushierarchien als Mittel der Legitimierung der Belohnungsunterschiede: materieller Wohlstand und Einkommen, Macht und Autorität. Die Verteilung des Bildungsstatus mag zur Rechtfertigung der Einkommensverteilung beitragen, d.h. Legitimität wird von der Bildung zum Einkommen übertragen. Diese Theorie ist von Peter Heintz formuliert worden ${ }^{4}$.

Die Grundidee bei der theoretischen Argumentation ist die Annahme, daß die Machtstruktur in einem sozialen System legitimiert werden muß, zumindest in gewissem Umfang. Der Bedarf an Legitimität bedeutet, daß eine legitimierende Statushierarchie - Bildung in der modemen Gesellschaft - einen legitimeren Wert darstellen muß als die zentrale Machtstruktur, die als Ursache für die Ungleichverteilung der gesellschaftlichen Belohnungen gesehen wird. Weiter müssen soziale Verknüpfungen zwischen Status in ausgegliederten Hierarchien bestehen, zwischen Bildung und Einkommen in dem hier in Rede stehenden Fall.

Eine der wichtigsten Quellen für die Legitimität eines gesellschaftlichen Wertes (die hier auch die ,inteme“ Legitimität genannt wird), ist das Aus$\mathrm{maB}$ der Gleichheit in der Verteilung von Mitgliedern einer Gesellschaft über die betreffende Statushierarchie, die den Zugang zu diesem Wert regelt. Je flacher und gleichmäßiger das Verteilungsprofil - also je größer der allgemeine $\mathrm{Zu}$.

4 Vgl. Heintz (1972a, 1972b, 1982), Bornschier und Heintz (1977). gang zu Status in der Statushierarchie -, desto größer ist die interne Legitimität ${ }^{5}$.

Das Bildungssystem als vergleichsweise legitimere und offenere Statushierarchie wird in dieser Theorie als ein Weg begriffen, auf dem die Statuserwerbswïnsche in berechtigte Ansprüche umgewandelt werden. In diesem Sinne spielt der Bildungserwerb die Rolle einer sozialen Investition. Der Prozeb der sozialen Legitimierung der Einkommensverteilung durch die Bildung auf der Ebene des Gesamtsystems fuhrt mithin über individuelle Statusinkonsistenzen zu legitimen Ansprüchen von Mitgliedern der $\mathrm{Ge}-$ sellschaft auf eine höhere und ausgeglichenere Teilnahme an den gesellschaftlichen Werten. Diese Ansprüche können in der Gesellschaft nur teilweise eingelöst werden, weil die intern weniger legitime und sozial mehr kontrollierte Belohnungshierarchie es verunmöglicht, daß alle individuellen Ansprüche auf Statusausgleich erfüllt werden können.

Die Vorhersage dieser Theorie betrifft zwei Dinge. Erstens führt der soziale Prozeß der Legitimierung zu einer gewissen Entsprechung des Status in der Bildungs- und Einkommenshierarchie. Zweitens ist diese Entsprechung weit davon entfernt, perfekt zu sein. Warum genau soll das der Fall sein? Diesen Punkt möchte ich noch etwas ausfuihren. Der Legitimierungsprozeß beinhaltet eine soziale Verknüpfungsnorm zwischen einem sozial legitimeren Wert als soziale Investition (Bildung) und einer intern weniger legitimen Belohnungsverteilung (Einkommen). In der sozialen Struktur kann die Bildung gemäß der Theorie nur dann eine Legitimierungsfunktion übernehmen, wenn sie tatsächlich legitimer ist als die Einkommensverteilung, die mehr von der Macht kontrolliert wird. Nur so kann Legitimität im sozialen Verknüpfungsprozeß übertragen werden; dies genau meint: Legitimierung.

Es ist nach der theoretischen Vorstellung nicht möglich, eine ungleiche Verteilung von Belohnungen aufrechtzuerhalten und diese gleichzeitig voll zu legitimieren, und zwar durch die Ubertragung von Ansprüchen aufgrund der formalen Bildung auf äquivalente Positionen beim Einkommen.

5 Weitere Quellen der Legitimität, die die Behandlung hier unnötig komplizieren würden, werden bei Bornschier und Heintz (1977: 36) angeführt. 
Dies hat folgenden Grund. Die Ubertragbarkeit von Legitimität setzt Unterschiede in der internen Legitimität - und damit in der Ungleichverteilung - und natürlich Verknüpfungsnormen voraus. Solche Ubertragungsprozesse finden auf dem individuellen Niveau im Verlauf der Statuszuweisung statt (Schule $\rightarrow$ Beruf $\rightarrow$ Einkommen). Selbst dann, wenn die Verknüpfungsnormen in dem Sinne perfekt wären, daß ein System sie auch faktisch optimal realisiert, kann bestenfalls eine gewisse Rangentsprechung zwischen Bildungs- und Einkommensstatus in der Gesellschaft entstehen, niemals aber für alle Mitglieder das gleiche Austauschverhältnis zwischen sozialen Investitionen und Belohnungen.

Daher ergibt sich auf jedem Statusniveau der formalen Bildung notwendigerweise eine Spannweite im Einkommen, und zwar auch dann, wenn die Verknüpfungsnorm optimal ist. Wird die Verknüpfungsnorm weniger stark beachtet, so ist die Spannweite größer. Die zwangsläufig entstehende Spannweite im Einkommen und damit die Beschränkung einer empirischen Korrelation werden mit der Graphik 1 dargestellt.

Bildung

(flachere Statuspyramide, intern legitimer)

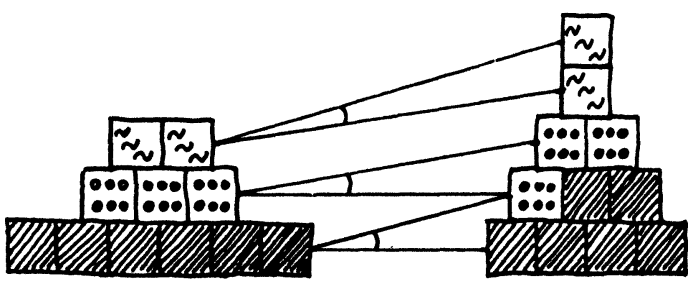

Symbolisiert die Optimierung der Rangkorrelation.

$\longrightarrow$ Symbolisiert die sich ergebende Spannweite im Einkommen.

GRAPHIK 1

In Graphik 1 sind die beiden Statuspyramiden, Bildung und Einkommen, in denen die Mitglieder einer Gesellschaft gleichzeitig einen Status einnehmen, schematisch dargestellt. Jedes Kästchen symbolisiert ein Vielfaches an Mitgliedern. Das Einkommen ist ungleicher verteilt (steilere Pyramide) und deshalb intern weniger legitim als die Bildung, die gleicher verteilt ist (flachere Pyramide) und deshalb ein intern legitimeres
Statussystem darstellt. Formale Bildung wird als eine Quelle für die Legitimierung des Einkommens aufgefaßt (Legitimität wird übertragen). Selbst wenn die Verknüpfungsnorm stark ausgeprägt ist, ergibt sich für jedes legitimierende Statusniveau der Bildung notwendigerweise eine Spannweite im Einkommensstatus.

Die Antwort dieser Theorie auf die zwei vorher gestellten Fragen lautet: Zwischen Bildung und Einkommen besteht eine positive Rangentsprechung, weil die Bildung die Funktion hat, die Einkommensverteilung zu legitimieren. Die Korrelation kann aber nicht vollkommen sein. Falls eine perfekte Korrelation bestünde (gleiche Austauschrate), würde die Bildung nicht die Funktion erfüllen, das Einkommen zu legitimieren, sie würde dann nämlich die gleiche Ungleichverteilung und mithin den gleichen Grad an interner Legitimität aufweisen wie die legitimationsbedürftige Belohnungsverteilung. Eine Ubertragung von Legitimität wäre ausgeschlossen.

\section{Merkmale der Legitimationstheorie}

Ein Vorteil der Legitimationstheorie besteht darin, daß sie auf beide hier in Rede stehenden Fragen eine Antwort geben kann. Ein Nachteil ist hingegen, daß die Theorie nicht im einzelnen ausführt, warum das Einkommen ungleich verteilt ist. Es wird zwar von der Machtkontrolle her argumentiert, aber die einschlägigen Mechanismen werden nicht ausgeführt.

Dieser Mangel kann allerdings behoben werden, wenn man andere Theorien, die zu diesem Punkt detaillierte Ausführungen machen, mit der Legitimationstheorie zusammenbaut.

Für die Legitimationstheorie ist charakteristisch, daß die Verknüpfung zwischen Bildung und Einkommen nicht inhaltlich hergeleitet wird. Dies ist verschieden von der Sichtweise der Bildung als Investitionsprozeß im engeren ökonomischen Sinne, bei dem Bildung Teil einer Produktionsfunktion wird. Bei der Legitimationstheorie hingegen wird die Verknüpfung als auf einer gesellschaftlichen Norm beruhend gesehen.

Dieser letzte Punkt ist insofern bedeutsam, als demzufolge bei einer Änderung der Bildungsverteilung keine dadurch bedingte gleiche Verände- 
rung der Einkommensverteilung erwartet wird. Stattdessen wird ein Wandel in der sozialen Verknüpfungsnorm zwischen Bildung und Einkommen vermutet, der in der Regel konfliktiv sein dürfte. Ein gewisser Nachteil einer solchen Auffassung, nach der die Verknüpfung allein aufgrund sozialer Normierung entsteht, ist der Umstand, daß Bildung auch inhaltlich, nämlich über eine Verbesserung der Arbeitsproduktivität, mit der Höhe des Einkommens verknüpft sein dürfte. Dies vernachlässigt die Legitimationstheorie, soweit sie bisher ausformuliert ist. Die Arbeitsteilungstheorie (Bornschier 1977, 1981) hat bei diesem Problem einen anderen Ansatz. Sie leitet die Struktur der Arbeitseinkommen wie auch die Struktur der im Arbeitsprozeß benötigten Fähigkeiten aus der Entwicklung der Arbeitsteilung her. Aber auch hierbei ist Bildung nicht notwendigerweise inhaltlich mit dem Einkommen verknüpft, vielmehr gilt dies nur in dem $\mathrm{Maße}$, wie die Verteilung der Bildungsqualifikationen mit derjenigen der Anforderungen an den Arbeitsplätzen übereinstimmen.

\section{Humankapital-Theorie}

In der Wirtschaftswissenschaft besteht eine lange bis auf Adam Smith zurückreichende Tradition, menschliche Fähigkeiten im Rahmen der Kapitaltheorie zu behandeln. Eine der jüngsten Entwicklungen in dieser Tradition ist eine allgemeine Theorie der Erklärung der Arbeitseinkommen aus dem Humankapital, die von Gary Becker, Jacob Mincer und ihren Schülern entwickelt worden ist ${ }^{6}$.

Im Zentrum dieses theoretischen Ansatzes steht die Vorstellung von Bildung, oder allgemeiner: Wissen, als einem Element in einer allgemeinen Produktionsfunktion. Bildung und Wissen, oder allgemeiner: menschliche Fähigkeiten, werden zu Kapital, nämlich Humankapital, im folgenden Sinne. Alles was die Produktivität der reinen Arbeit, die nur die Anwendung der natürlichen menschlichen Fähigkeiten beinhaltet, verbessert, wird als Kapital aufgefaßt.

Die Humankapital-Theorie der Arbeitseinkommen spezifiziert dann eine Einkommensfunktion,

6 Vgl. Becker (1964), Becker und Chiswick (1966), Mincer (1974, 1976). bei der die Verdienste einmal eine Funktion des Lohnes sind, den jemand ohne jegliche Investitionen in Humankapital erzielen würde; hinzu kommen die Erträge aus früheren Investitionen in Humankapital. Fähigkeiten zu erwerben bedeutet Ausbildung und Übung sowie einen späteren Eintritt in das Arbeitsleben. Das dadurch verpaßte Einkommen und der Aufwand werden später zurïckbezahlt in Form von Erträgen auf Humankapital-Investitionen.

Um zu erklären, wieviel Investitionen in Humankapital getätigt werden, postuliert die Theorie ein Optimierungsverhalten von seiten der Individuen (oder ihrer Eltern). Die Entscheidung, in sich selbst zu investieren, gründet auf dem gegenwärtigen diskontierten Ertrag von verschiedenen Mengen an Humankapital-Investitionen. Das bedeutet in den Worten Atkinsons (1975: 81; meine Übersetzung - V.B.: ,damit eine Person indifferent ist, ob sie die Schule zum Zeitpunkt des Ablaufs der gesetzlichen Schulpflicht verläßt oder sich weiter für einen Arbeitsplatz ausb ildet, der soundsoviel Jahre weitere Ausbildung erfordert, muß der diskontierte Gegenwartswert der Verdienste gleich sein."

Die formale Schulbildung umfaßt nicht alle Investitionen in Wissen und Fähigkeit. Es gibt u.a. noch: Ausbildung nach der Schule, Ubung im Arbeitsleben und Lernen am Arbeitsplatz. Dennoch steht die formale Schulbildung wegen der leichten Meßbarkeit im Mittelpunkt der Untersuchungen von Forschern, die mit dem Humankapital-Ansatz arbeiten (vgl. Sahota 1978). In empirischen Studien wird gewöhnlich die Länge der formalen Schulbildung als Maß für das Humankapital verwendet, was eine nicht adäquate Operationalisierung ist.

Zusammenfassend kann man sagen: Die Humankapital-Theorie macht die Vorhersage, daß die Verdienstunterschiede vom Ausmaß der nötigen Ausbildung abhängen. Und diese Verdienstunterschiede sind gerade groß genug, um die Kosten für diese Ausbildung zurückzuzahlen. Ein solches Gleichgewicht en twickelt sich durch den Lohnwettbewerb auf dem Arbeitsmarkt, den die Theorie explizit unterstellt. Ungleichgewichte im Sinne von zu hohen Investitionen in Humankapital bei einer bestimmten Nachfrage nach $\mathrm{Ar}$ beitskräften werden über den kompetitiven $\mathrm{Ar}$ beitsmarkt (längerfristig) ausgeglichen. Wie Sahota (1978: 13) richtig bemerkt, beachtet eine 
solche Theorie nicht die Segmentierung des Arbeitsmarktes; dieser zerfällt in verschiedene Teilmärkte für Arbeitskräfte, die untereinander nicht in Wettbewerb stehen (principle of noncompeting groups), eine Vorstellung, die ebenfalls auf eine lange Tradition zurïckblicken kann.

Die Antworten der Humankapital-Theorie auf die beiden gestellten Fragen fallen folgendermaBen aus. Erstens besteht zwischen Bildung und Arbeitseinkommen eine Entsprechung, weil sich die Verdienste aus den Humankapital-Investitionen herleiten. Zweitens wird eine perfekte Entsprechung erwartet. Dies ist ein wichtiger Unterschied in der Vorhersage zwischen der HumankapitalTheorie auf der einen Seite und der Legitimationstheorie sowie den weiter unten zu behandelnden Auswahltheorien andererseits.

\section{Merkmale der Humankapital-Theorie}

Die Tatsache einer nur mäßigen gemeinsamen Varianz zwischen Bildung und Arbeitseinkommen kann man kritisch gegen den empirischen Erklärungsgehalt der Theorie vorbringen. Allerdings umfaßt das Konzept „Humankapital“ mehr als die Länge der formalen Schulbildung. Dementsprechend verbessert z.B. die Berücksichtigung der Ausbildung nach der Schule die Erklärung der Verdienstunterschiede. Die empirische Erklärung bleibt aber dennoch ziemlich schwach. In den Arbeiten von Jacob Mincer können dann nur rund ein Drittel der Unterschiede beim Arbeitseinkommen erklärt werden (Sahota 1978: 13).

Selbst wenn zusätzliche Annahmen in das Erklärungsmodell eingebaut werden, wie eine höhere Elastizität der Bildungsinvestitionen nach der Schule und eine positive Beziehung zwischen erreichtem Schulabschluß und Arbeitseinsatz - Annahmen, die selbst nicht aus dem Humankapital-Ansatz folgen -, bleibt die empirische Erklärung der Verdienstunterschiede unbefriedigend, jedenfalls gemessen am theoretischen Anspruch. Dann kann zusammen die Hälfte der Ungleichheit bei den beobachteten Arbeitseinkommen auf die Bildung während und nach der Schule zurückgefuihrt werden. Solche zusätzlichen Annahmen erscheinen mir aber insofern problematisch, als man den Arbeitseinsatz nicht unter den Begriff des Humankapitals einordnen kann.
Und eine höhere Elastizität des nach der Schule erworbenen Humankapitals dürfte Folge von organisationellen Faktoren sein, nämlich u.a. Folge von Aufstieg in Organisationen, über den die Arbeitgeber entscheiden.

Dennoch könnte man zur Verteidigung des Ansatzes einwenden, daß die gemeinsame Varianz zwischen Humankapital und Verdiensten, wie man sie bisher in empirischen Studien ermittelt hat, zu falschen Schluissen verleite und solche Untersuchungsanordnungen nicht die Idee der Theorie voll repräsentieren, denn nicht aktuelle Jahresverdienste, sondern die über das Leben kumulierten Verdienste müßten verglichen werden.

Die Erklärung auf der Grundlage des Humankapitals ist meistens verwendet worden, um die Unterschiede im Arbeitseinkommen zwischen Personen vorherzusagen. Lydall (1976: 22) hat gegen die Theorie deshalb eingewendet, sie könne nicht das bekannte Muster der schiefen Gesamtverteilung der Arbeitseinkommen erklären, weil sie nicht voraussagen könne, warum bestimmte Personen mehr in sich investieren als andere.

Diese Behauptung stimmt nicht ganz. Man mag zwar die Erklärung der gesamten Ungleichheit der Arbeitseinkommen als problematisch empfinden, aber es gibt solche (Sahota 1978). Z.B. argumentiert Becker (1964) folgendermaßen. Der Grund für die schiefe Verteilung der Verdienste ist, daß Personen mit höheren Fähigkeiten einen größeren Ertrag aus ihrem Humankapital beziehen und deshalb mehr in sich investieren. Eine damit unterstellte multiplikative Beziehung erzeugt eine schiefe Verteilung des Humankapitals (selbst wenn die natürlichen Fähigkeiten normalverteilt wären), die nach Becker der schiefen Verteilung der Arbeitseinkommen entspricht. In der Graphik 2 stelle ich diese Argumentation dar.

Tatsächlich kann man die mit dem Argument unterstellte positive Beziehung zwischen (natürlichen) Fähigkeiten und der Länge des Schulbesuchs empirisch aufweisen (eine Korrelation von mäßiger Höhe). Aber Lydall (1976: 23) macht berechtigterweise den polemischen Einwand: „Zu behaupten, daß die Korrelation (zwischen Fähig. keit und Schulbesuch, V.B.) sich allein aus den 


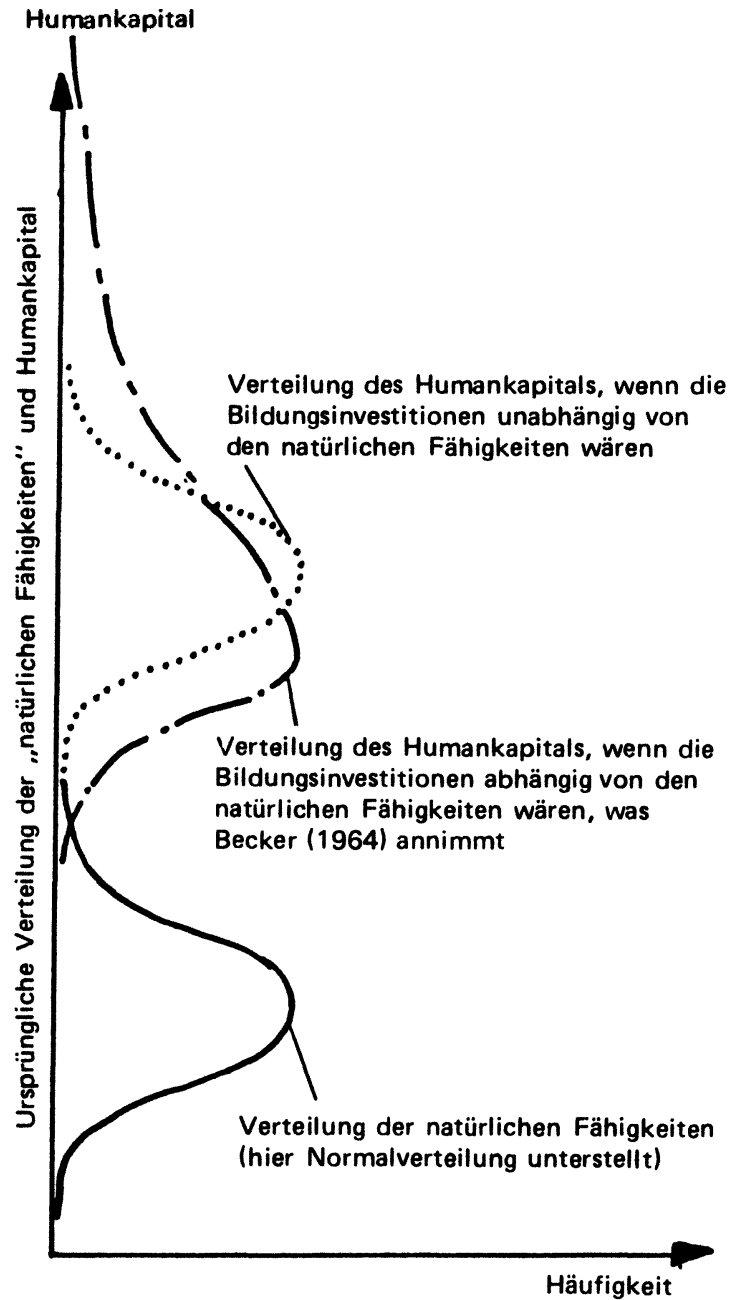

GRAPHIK 2

profitmaximierenden Entscheidungen von Investoren in Humankapital herleite, bedeutet sicherlich, $\mathrm{da}$ man all jene naheliegenden Gründe ignoriert, die eine positive Korrelation zwischen Fähigkeit und Schulbesuch bewirken. Gibt es denn kein Prüfungssystem an der Columbia University?"

Man kann mithin einwenden, daß das Humankapital-Modell die Einflüsse des Schulsystems und der sozialen Herkunft unberücksichtigt lasse. Diese Faktoren sind u.a. von großer Bedeutung bei der soziologischen Erklärung der Ungleichheit. Eine „Erbtheorie“" stellt z.B. den Umstand heraus, $d a ß$ die Schulbildung einer der wichtigsten Wege in der modernen Gesellschaft ist, den sozialen Status von einer Generation auf die nächste zu übertragen, einmal weil der Zugang zur höheren Schulbildung von der sozialen Lage der Herkunftsfamilie abhängt und weil zudem in der modernen Gesellschaft eine normative Verknüpfung zwischen erreichter Schulbildung und dem Anspruch auf den Zugang zu hochbewerteten gesellschaftlichen Gütern besteht.

Weiter spielen die Arbeitgeber keine Rolle. Sie aber bestimmen die Art und den Umfang des Humankapitals, das sie bereit sind, gegen Bezahlung im Arbeitsprozeß zu verwerten (vgl. zu diesem Punkt auch Reder 1969). Die einzige Bezugnahme auf den Arbeitsmarkt steckt in der Annahme eines Lohnwettbewerbs. Schließlich ist anzumerken, daß die Humankapital-Theorie trotz ihrer Prominenz im modernen sozialwissenschaftlichen Denken nur eine Teilerklärung sein kann. Sie beschränkt sich darauf, die Arbeitseinkommen zu erklären. Die Einkommenskapazität aufgrund von Vermögen sowie die Verwendung von Vermögen und Humankapital als Arbeitgeber oder Selbständiger bleiben außerhalb der Reichweite der Theorie.

Es bleibt noch anzumerken, daß die Humankapital-Theorie im Gegensatz zur Legitimationstheorie und Auswahltheorie (siehe weiter unten) voraussagt, daß die Verteilung der Arbeitseinkommen sich notwendigerweise nach Maßgabe einer geänderten Verteilung des Bildungskapitals verändert. Dies ist ein wichtiger Unterschied in der Prognose.

\section{Auswahltheorien der Bildung}

Die beiden Theorien, die ich unter der Hauptüberschrift „Auswahltheorien" darstelle, berücksichtigen die Struktur der Arbeitsplätze und das Arbeitgeberverhalten bei der Herleitung der Verknüpfung zwischen Bildung und Einkommen in der modernen Gesellschaft. Insoweit die Arbeitgeber beim Auswahlprozeß die Legitimierung der geschichteten Arbeitsplätze und die aus der Bildung abgeleiteten Ansprüche von Stellenbewerbern berücksichtigen, sind Auswahltheorien leicht mit der im Hinblick auf das Arbeitgeberverhalten unspezifizierten Legitimationstheorie zu verbinden. Kaum verknüpfbar sind Auswahltheorien allerdings mit der Humankapital-Theorie, bei der die Investitionen in Bildung 
einmal aufgrund individueller Wahlentscheidungen zustande kommen und sich dann gleichsam automatisch - vermittelt nur über einen Lohnwettbewerb auf dem Arbeitsmarkt - in ein Arbeitseinkommen umsetzen, ohne daß dem Arbeitgeber dabei eine besondere Rolle zukäme.

Den Auswahltheorien der Bildung ist gemeinsam, daß individuellen Merkmalen im allgemeinen und der Bildung im besonderen im wesentlichen nur Signalwert für den Arbeitgeber zukommt. Nicht die formale Bildung als solche, sondern ihr Signalwert für die Einschätzung der internen Ausbildungskosten bei Lester Thurow $(1975,1978)$ oder für legitime Ansprüche und für die Wahrscheinlichkeit der Produktivität und Konformität einer betreffenden Person (Bornschier 1977, 1981) steht dabei im Vordergrund der theoretischen Argumentation.

Beide Versionen einer Auswahltheorie erklären die unvollkommene Korrelation zwischen formaler Bildung und der Höhe der Verdienste. Es wird begründet, daß diese Korrelation gar nicht vollkommen sein kann. Dies ist die gleiche Schlußfolgerung wie die der Legitimationstheorie, die sich klar abhebt von der Erwartung der Humankapital-Theorie.

\section{Arbeitsteilung und Bildung als Auswahlhilfe- verfahren}

Die Arbeitsteilungstheorie leitet die Struktur der Arbeitseinkommen aus den Entwicklungen im Verlauf der organisationellen Evolution ab. $\mathrm{Zu}$ verschiedenen Zeitpunkten des evolutionären Prozesses ergeben sich bestimmte Muster der Verteilung der Personen auf bestimmte Arbeitsplätze in der Gesellschaft, die sich aus der Aggregierung der einzelnen organisationellen Verteilungsprofile ergeben (Bornschier 1977, 1981).

Der Kernpunkt der Theorie betrifft die Vorstellung einer zunehmenden dreifachen Gabelung der Arbeit im Verlauf der Entfaltung der Arbeitsteilung in: Routinearbeit, Expertenarbeit und Kontrollarbeit. Die drei entsprechenden Prozesse: Routinisierung, Spezialisierung und Hierarchisierung bilden einen Systemzusammenhang. Die wirtschaftlichen Vorteile der Arbeitsteilung liegen in den Ersparnissen begründet, die die Routinisierung ermöglicht. Wenn Organisationen expandieren wollen, so sind diese Ersparnisse Voraussetzung für den Ausbau der Hierarchie, der seinerseits notwendig ist, um den Kontrollverlust der letzten Herrschaft abzuwenden, und für die Zunahme der Zahl und der Qualifikation von Experten, die der durch die Hierarchie verkörperten Herrschaft zur Seite stehen müssen, um die Probleme der komplexer werdenden Organisationen in einer vielgestaltigen organisationellen Umwelt zu lösen.

In diesem Prozeß kann Humankapital überhaupt gesamtgesellschaftlich von den Organisatoren, Verwaltern und Spezialisten in wachsendem Maße erworben und im Arbeitsproze $\beta$ angewandt werden, weil die mit Routinearbeit betrauten Arbeitskräfte zunehmend von angewandten Fähigkeiten im Arbeitsprozeß entkleidet werden. Andererseits sparen die höhere Produktivität und die verbesserte Technik Routinearbeiter ein. Diese personellen Ersparnisse sind notwendig, um die wachsende Zahl der Positionen in der expandierenen Hierarchie zu besetzen. Und die Hierarchie braucht auf allen Stufen zunehmend Hilfe von Experten, damit die Folgen der Entdifferenzierung der Arbeit aufgefangen werden können. Diese hier nur kurz skizzierten Prozesse beinhalten, daß sich zwei unterscheidbare Aspekte der Ungleichheit gegensätzlich entwikkeln: Die positionale Ungleichheit nimmt mit der Entfaltung der Arbeitsteilung zu (Hierarchisierung der Arbeitsrollen), aber die distributive Ungleichheit verringert sich (der Modus der Häufigkeit der Stellenbesetzung verschiebt sich im Beschäftigungssystem etwas nach oben $)^{7}$.

Die Theorie argumentiert, daß sich die Struktur der Arbeitsplätze mit der korrespondierenden Verteilung der bei der Arbeit angewendeten Fähigkeiten aus der Entfaltung der Arbeitsteilung herleitet und nicht aus dem Bildungsangebot der Arbeitskräfte. Die Struktur und Ungleichheit der Löhne und Gehälter sind nach dieser Theorie abhängig von der Arbeitsteilung. Oberhalb des Grundlohnes für Routinearbeit, der sich aus einem Lohnwettbewerb ergibt, ist die Lohnund Gehaltsabstufung abhängig von der Macht der Positionen in der Hierarchie. Die sich daraus ergebende aggregierte Verteilung der Arbeitsein-

7 Auf strukturelle Arbeitslosigkeit und Unterbeschäftigung bei raschem technischen Fortschritt gehe ich hier nicht ein, vgl. Bornschier $(1977,1981)$. 
kommen ist dementsprechend sehr schief, eine Begründung die sehr verwandt ist mit derjenigen, die Lydall (1968) mit seinem Modell der ,hierarchischen Arbeitseinkommen" gibt.

\section{Bildung und Arbeitseinkommen sind nach der} Arbeitsteilungstheorie folgendermaßen verbun. den. Das latente Arbeitskräfteangebot für höherrangige, mithin privilegierte Positionen in der Hierarchie der Arbeitsplätze ist größer als die effektive Nachfrage. Deswegen können die Arbeitgeber eine Auswahl treffen. Die formale Bildung ist nur ein Kriterium unter anderen bei der Auswahl, ein - wenn man so will - universalistisches Auswahlhilfeverfahren, das auch den Legitimierungsproze $ß$ berücksichtigt und für den Arbeitgeber zudem den Vorteil hat, fast zum Nulltarif zur Verfugung zu stehen. Hinzu kommen mehr partikularistische Kriterien bei der Auswahl auf verschiedenen Stufen der externen Rekrutierung und beim Prozeß der Beförderung.

Die partikularistischen Kriterien sind insbesondere: die Konformität mit den Zielen der betreffenden Organisation und die Fähigkeit sowie der Wille, die Zielerreichung der Organisation voranzutreiben und die Machthierarchie zu stützen. Abgesehen von den untersten Stufen der Hierarchie bedeuten Anstellung und Beförderung eine Kooptierung der Betreffenden in die Machtstruktur der Organisation. Diese Kooptierung gründet - wie erwähnt - nicht ausschließlich auf der formalen Bildung und materialisiert sich in einem höheren sozialen Status und höheren materiellen Belohnungen ${ }^{8}$.

Bei einer solchen kombinierten Selektion durch Arbeitgeber wird die Verteilung der kombinierten Merkmale der Beschäftigten, die dem Bedarf der Organisation an Untermauerung der

8 Zudem liefert ein hoher sozialer Herkunftsstatus in der Regel u.a. auch eine detaillierte Vorsozialisierung in bezug auf die Werte und Normen, die auf dem externen und internen Arbeitsmarkt für gehobene Berufspositionen Konkurrenzvorteile im späteren Arbeitsleben verschaffen. Deswegen dürfte die soziale Herkunft auch neben der formalen Bildung und dem Willen zur Konformität einen unabhängigen Einfluß auf den Zugang zu gehobenen Positionen im Beschäftigungssystem haben, der sich aber erst im Verlauf der beruflichen Karriere voll entfalten dürfte. Zum sogenannten Späteffekt der Herkunft vgl. auch Bornschier (1977).
Struktur der Arbeitsteilung entspricht, stark ungleichverteilt, selbst wenn die formale Bildung normalverteilt wäre. Und diese ungleichverteilte Kombinationseigenschaft entspricht der Verteilung der Löhne und Gehälter. Obwohl die Bildung ein wichtiges Element der kombinierten Selektion darstellt, kann diese dennoch nicht stark mit den Unterschieden bei den Löhnen und Gehältern assoziiert sein. Dies ist die Antwort der Theorie auf die beiden gestellten Fra. gen.

Man kann die Theorie noch dadurch verfeinern, daß man explizit zwischen externen und internen Arbeitsmärkten unterscheidet. Auf bestimmten Stufen der Hierarchie werden die Arbeitskräfte vornehmlich extern rekrutiert, während zwischen solchen Stufen die Arbeitsplätze hauptsächlich durch Beförderung besetzt werden. Die interne Beförderung ist ziemlich unabhängig von der Auswahlhilfefunktion der formalen Bildung, weil dieses Auswahlverfahren bereits an den verschiedenen externen Rekrutierungsstellen angewendet wurde. Aus der Tatsache, daß von jeder Stufe nur ein Teil befördert wird und daß diese Personen unterschiedlich weit in der Hierarchie aufsteigen, ergibt sich zusätzlich eine erhebliche Spannweite in der Höhe der Löhne und Gehälter, die jeweils einem formalen Bildungsniveau entsprechen. Und der Umstand, daß auf jeder externen Rekrutierungsstufe die formale Bildung nur ein Selektionskriterium darstellt, bewirkt, daß selbst die Rangentsprechung zwischen formaler Bildung und Arbeitseinkommen bei weitem nicht perfekt ist.

Von allen hier in Rede stehenden Theorien erwartet deshalb die Arbeitsteilungstheorie die geringste Korrelation zwischen formaler Bildung und Verdienst. Zwar ist die diesbezügliche Aussage der Legitimationstheorie abhängig von der Stärke der sozialen Verknüpfungsnorm, aber die Arbeitsteilungstheorie impliziert, daß sich diese Norm faktisch nicht über eine bestimmte Stärke hinaus bilden wird, da die Arbeitgeber zusätzlich zur Bildung nach weiteren Kriterien selektieren.

Die Tatsache, daß in der modernen Gesellschaft eine ausgeprägte Segmentierung der Organisationen nach Maßgabe der Entwicklung der Arbeitsteilung besteht, verändert die Aussage der Theorie nicht entscheidend. Zwar können die führen- 
den Organisationen infolge ihrer größeren Zahlungsfähigkeit auf allen Rekrutierungsstufen höhere Bildungsqualifikationen als Eintrittsbedingungen festlegen, aber dafür zahlen sie auch für vergleichbare Arbeitsplätze mehr als kleinere Organisationen (Bornschier 1976: $293 \mathrm{ff}$.), weswegen sich die Beziehung zwischen Bildung und Verdienst dadurch gesamthaft kaum wesentlich ändert. Auch das stärkere Gewicht, das die mit Blick auf die Arbeitsteilung fortgeschritteneren, größeren Organisationen wegen ihrer längeren Hierarchien auf die interne Rekrutierung legen (wodurch sich - für sich betrachtet - die Verknüpfung abschwächt), wird kompensiert durch das größere Gewicht, das normalerweise die Großorganisationen auf die formale Bildung bei der externen Rekrutierung legen. Kleinere Organisationen selektieren wohl bereits stärker bei der Anstellung zusätzlich zur Bildung, während dies bei den großen vermehrt erst im Verlauf des Aufstiegs geschieht. Und dieser potentielle Aufstieg ist viel länger und mithin die dadurch bedingte Lockerung der Verknüpfung größer als bei kleinen Organisationen.

Die Graphik 3 erklärt die theoretischen Zusammenhänge vereinfachend. Die externe Rekrutierung auf jeder Eintrittstufe gründet nicht allein auf der formalen Bildung, sondern auch auf zusätzlichen Selektionskriterien (Konformität usw., vgl. weiter oben). Jene Stellenbewerber, die angestellt werden, erfüllen die zusätzlichen Bedingungen (symbolisiert durch die Punkte in den Kästchen), die übrigen mit der gleichen formalen Bildung müssen sich auf tieferen Eintrittsstufen bewerben oder in ihren alten Beschäftigungsverhältnissen verbleiben. Die Beförderung stützt sich nicht mehr wesentlich auf die formale Bildung; diese war bereits Selektionskriterium bei der Anstellung. Und auf jeder Stufe, von der aus die interne Beförderung ihren Ausgang nimmt, haben die Beschäftigten ein vergleichbares formales Bildungsniveau. Das Ergebnis des Modells ist eine nur mäßige Korrelation zwischen formaler Bildung und dem Arbeitseinkommen, mithin eine erhebliche Spannweite im Verdienst für gleiche formale Bildungsniveaus.

2. Arbeitsplatzwettbewerbs-, Arbeitskräftewarteschlangen- und Indikatortheorie der Bildung

Die letzte zu behandelnde Theorie ist von Lester Thurow $(1975,1978)$ vorgeschlagen worden
Stellenbewerber mit vier Stufen formeller Bildungsabschlüsse

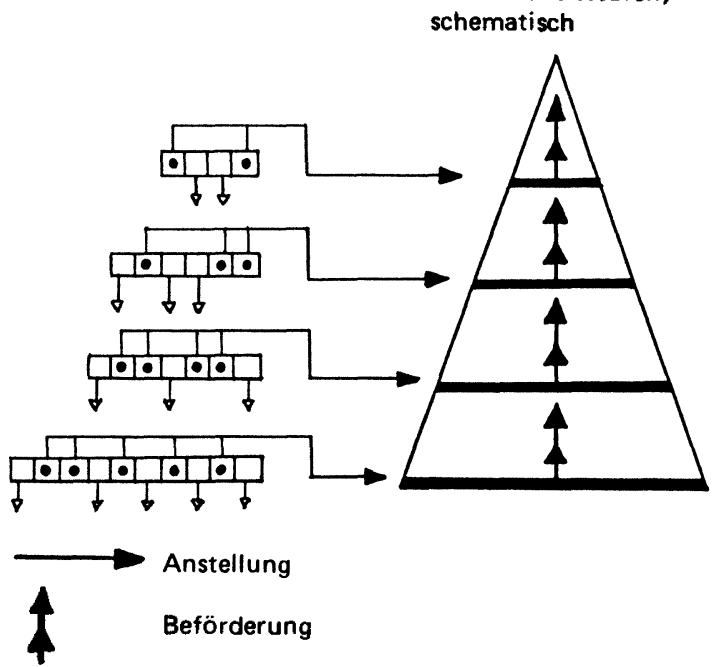

Bewerbung für andere Arbeitsplätze oder Verbleiben am momentanen Arbeitsplatz

GRAPHIK 3

und weicht in wesentlichen Punkten von den üblichen Annahmen in der ökonomischen Theorie $a b$. Zunächst einmal gründet die Theorie nicht auf der Annahme eines Lohnwettbewerbs. Stattdessen konkurrieren Arbeitskräfte in ,Warteschlangen" eher um Arbeitsplätze, zumindestens, wie Lester Thurow argumentiert, in vielen Industrien. Weiter postuliert die Theorie, daß die interne Firmenausbildung die entscheidende Bestimmungsgröße für die Produktivität von Arbeitskräften ist. Um produktive Arbeitskräfte zu erhalten, haben Arbeitgeber deshalb Investitionen in Ausbildung zu tätigen. Der Wettbewerb um Arbeitsplätze wird in Thurows Sichtweise damit gleichbedeutend mit: Wettbewerb um firmeninterne Ausbildungsplätze.

Da Arbeitgeber ihre Kosten generell tief halten wollen und die firmeninteme Ausbildung erhebliche Kosten verursacht, suchen sie nach einem Indikator, der ihnen eine Schätzung ihrer Ausbildungskosten für verschiedene Stellenbewerber ermöglicht. Dies muß ein allgemein anwendbarer und valider Indikator ohne große Kosten sein, der erlaubt, jeden Stellenbewerber gemäß den geschätzten internen Ausbildungskosten zu rangieren, Kosten, die nötig sind, um einen Stellenbewerber am Arbeitsplatz produktiv zu machen. 
Mit anderen Worten: Die Arbeitgeber brauchen einen Indikator für die Ausbildungsfähigkeit. Je höher die Ausbildungsfähigkeit eines Kandidaten, desto tiefer sind die Kosten der Arbeitgeber für einen betreffenden Arbeitsplatz.

Die Theorie arbeitet mit der Vorstellung einer Warteschlage von Stellenbewerbern vor den Arbeitsplätzen. Diese Warteschlange ist von den $\mathrm{Ar}$ beitgebern geordnet worden, von den geringsten bis hin zu den höchsten internen Ausbildungskosten. Der zentrale Indikator für die Ausbildungsfähigkeit ist die erreichte Schulbildung (man könnte hier präzisieren: „Schulleistung“, also Schulbildung gemessen an den dafür benötigten Jahren). Ein Stellenbewerber mit besserer Schulbildung hat bewiesen, daß seine Ausbildungsfähigkeit groß ist. Kognitive Fähigkeiten, Fleiß und Disziplin sind ebenfalls Korrelate des Schulerfolgs. Deshalb ist die formale Schulbildung ein guter Sammelindikator für die internen Ausbildungskosten, der zudem noch zum Nulltarif zur Verfügung steht.

Aus der nach Ausbildungsfähigkeit geordneten Warteschlange greifen die Arbeitgeber so viele heraus, wie es für die Besetzung ihrer offenen Stellen nötig ist. Diejenigen, die eine Anstellung erhalten, werden intern ausgebildet und dadurch für den Arbeitgeber einsetzbar. Jene, die nicht aus der Warteschlange herausgegriffen werden, weil sie rangmäßig zu weit hinten stehen oder weil zu viele mit den gleichen Ausbildungschancen auf gleicher Höhe in der Schlange stehen, müssen an ihren alten Arbeitsplätzen verbleiben oder sind arbeitslos. Dies wird mit der Graphik 4 anschaulich dargestellt.
Arbeitskräftewarteschlange nach Ausbildungsfähigkeit geordnet gemäß dem Indidung kator: formale Schulbilplätze auf einem bestimmten Niveau und bei einem bestimmten Arbeitgeber
Zu besetzende Arbeits-

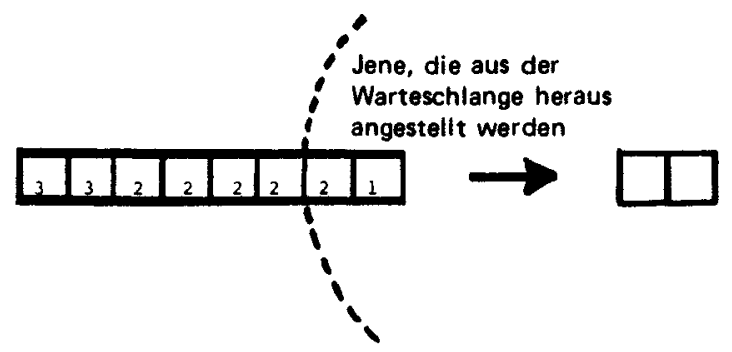

Die Indikatortheorie erklärt eine gewisse Entsprechung zwischen formaler Bildung und der Höhe der Löhne und Gehälter. Diese Korrelation ist aber unvollkommen, weil die interne Ausbildung der Grund für die Arbeitsproduktivität ist, die den Lohn sowie die Beförderung bestimmt. Die formale Bildung ist demnach nur ein anfänglicher Indikator für die Arbeitgeber bei der Anstellung. Die Arbeitseinkommen ergeben sich aufgrund der Bewertung der Arbeitsplätze und der internen Ausbildung und werden deshalb im Verlauf der beruflichen Karriere zunehmend unabhängiger vom ursprünglichen Indikator für die Ausbildungsfähigkeit.

Wie bei der Arbeitsteilungstheorie erhalten auch nach dieser Theorie Stellenbewerber mit der gleichen formalen Bildung nicht immer das gleiche Anfangsgehalt. Während der Grund bei der Arbeitsteilungstheorie in der zusätzlichen Selektion gemäß der Konformität usw. liegt, ist es nach der Theorie der Arbeitskräftewarteschlange der Bedarf der Arbeitgeber an Arbeitskräften, was vom Bildungsangebot (Indikator für die Ausbildungsfähigkeit) ziemlich unabhängig sein kann.

Die zuletzt behandelte Theorie kann mithin die Einflüsse der Konjunktur auf die Beziehung zwischen Bildung und Arbeitseinkommen einbeziehen, und zwar mit der Voraussage, daß sich durch konjunkturelle Höhen und Tiefen auf dem Arbeitsmarkt die in Rede stehende Verknüpfung abermals lockert. Wenn Arbeitgeber mehr Arbeitskräfte einstellen, weil die Konjunkturaussichten günstig sind, dann greifen sie tiefer in die Warteschlange der Arbeitskräfte hinein, bei ungünstigen Aussichten weniger weit. Da das $\mathrm{Kri}$ terium für die Rangierung der Warteschlange aber gleich bleibt, ist es erheblich, wann sich jemand auf dem Arbeitsmarkt um eine Stelle bemüht. Bedingung für diese Argumentation ist die Annahme der Theorie, daß erst die interne Ausbildung die Produktivität am Arbeitsplatz schafft.

Durch die interne Ausbildung können die unterschiedlich entstehenden Verknüpfungen zwischen formaler Bildung (Indikator für Ausbildungsfähig keit) und Arbeitseinkommen nur sehr beschränkt durch Lohnwettbewerb ausgeglichen werden. Hat z.B. jemand in Zeiten der Hochkonjunktur trotz vergleichweise tiefer formaler Bildung einen firmeninternen Ausbildungsplatz erhalten, dann schützen ihn die Ausbildungskosten der Firma später in gewissem Ausmab vor Wettbewerb, 
auch von seiten von Stellenbewerbern mit höherer formaler Bildung, selbst wenn diese bereit sind, zu einem niedrigeren Lohn zu arbeiten. Die spezifische, firmeninterne Ausbildung schränkt mithin die Flexibilität des Arbeitsmarktes ein, auch bindet sie die Arbeitskräfte eher an einen bestimmten Arbeitgeber, da das intern erworbene Ausbildungskapital häufig nur beschränkt zwischen Arbeitgebern übertragen werden kann (Reder 1969). Ist es leicht übertragbar, so sind die Arbeitgeber gezwungen, „Treueprämien“ zwecks Vermeidung von Stellenwechsel zu zahlen.

\section{Abschließende Merkmale der Auswahltheorien der Bildung}

Bei der Theorie der Warteschlange der Arbeitskräfte bestehen Berührungspunkte mit der Vorstellung eines internen Arbeitsmarktes bei der Arbeitsteilungstheorie und mit der Kategorie der Ausbildung am Arbeitsplatz bei der Humankapitaltheorie. Die letztgenannte Nähe erscheint größer, als sie es tatsächlich ist. Denn im Gegensatz zur Humankapital-Theorie bestimmen bei der Auswahltheorie nicht individuelle Wahlhandlungen, sondern Entscheidungen von Arbeitgebern, ob und wieviel produktives Humankapital investiert wird. Und dies hängt davon $a b$, wieviel davon Arbeitgeber benötigen und zu finanzieren gewillt sind.

Ein Vorteil der Auswahltheorien der Verknüpfung zwischen Bildung und Einkommen liegt in der Berücksichtigung des Arbeitgebers, der Struktur der Arbeitsplätze und der ausgeführten Vorstellungen über den Verknüpfungsprozeß. Der leicht verallgemeinerungsfähige Charakter der Auswahlvorstellung ist ebenfalls von Vorteil. Die formale Bildung mag einen hohen Stellenwert bei der Selektion haben oder ihr mag ein hoher Indikatorwert zukommen, aber es muß nicht ausschließlich die formale Bildung sein, nach der die Arbeitgeber auswählen. Dies ist wichtig für eine Erklärung der Tatsache, daß etliche weitere soziale Merkmale mit der Höhe des Einkommens ebenfalls in Zusammenhang stehen.

Die Arbeitgeber mit großer Zahlungsfähigkeit können die höchsten Löhne und Gehälter für bestimmte Arbeitsplätze bieten und dafür die Stel- lenbewerber mit sozial bevorzugten Merkmalen anstellen, wie z.B. Ethnie oder Rasse, Geschlecht, soziale Herkunft, Alter, Zivilstand, äußere Erscheinung und „Manieren“ usw. Solche sozialen Merkmale dürften einem gewissen Konsensus über die Legitimität der sozialen Schichtung von Belohnungen entsprechen, und solche zusätzlichen Verknüpfungen mögen dann zur Legitimierung der Macht- und Einkommensstruktur beitragen.

\section{Schlußbemerkungen}

Dieser Aufsatz hat sich zum Ziel gesetzt, verschiedene theoretische Argumente kurz darzustellen, die für das Verhältnis von Bildung und Verdiensten in der modernen Gesellschaft bedeutsam sind. Eine erschöpfende Darstellung aller möglichen Argumente war nicht vorgesehen. Schon die vier vorgestellten Argumentationsfiguren decken einen weiten Bereich unterschiedlicher Sichtweisen ab und mögen unsere Theoriebildung sowie die theoretische Integration stimulieren wie auch unsere empirischen Forschungen durch Arbeitshypothesen vorantreiben.

Drei von den vier behandelten Theorien argumentieren, daß der Zusammenhang zwischen Bildung und Arbeitseinkommen in der Gesellschaft aus theoretischen Gründen nicht besonders hoch sein kann. Die empirischen Evidenzen zur gemeinsamen Varianz stützen eine solche Sichtweise. Nur die Humankapital-Theorie sagt eine perfekte Ubereinstimmung zwischen der Verteilung der Verdienste und des Humankapitals voraus. Aber auch Forscher, die mit diesem theoretischen Rahmen arbeiten, haben nie eine empirische Erklärung erreicht, die über ein Drittel an gemeinsamer Varianz hinausgeht.

\section{Die Verknüpfung von Bildung und Einkommen} ist hier Gegenstand gewesen. Deshalb wurden Einzelheiten der Verteilungsprofile von Bildung und Einkommen nicht behandelt. Die Theorien machen sehr unterschiedliche Voraussagen über die Folgen einer Änderung der Bildungsverteilung. Drei Theorien behaupten, daß eine Änderung der Bildungsverteilung keine direkten Auswirkungen auf die Einkommensverteilung habe ${ }^{9}$,

9 Die Bildung mag indirekte Auswirkungen auf die Einkommensverteilung (vgl. Anmerkung 1) haben, z.B. vermittelt über politische Prozesse, die Einkommensumverteilungen zur Folge haben. 
während die vierte Theorie eine Änderung bei der Verteilung der Arbeitseinkommen behauptet, die genau der Änderung in der Verteilung des Humankapitals entspricht. Die empirischen Befunde zur Änderung in der Verteilung der Einkommen und der Verdienste lassen wegen der unvollständigen Datenlage keine ganz sicheren Schlüsse zu. Aber zumindest in den sechziger und siebziger Jahren war die Einkommensverteilung in den wesentlichen Industrieländern durch eine bemerkenswerte Stabilität gekennzeichnet, obwohl sich gerade in dieser Zeit die Struktur der Bildung in der Bevölkerung merklich verändert haben dürfte. Es wäre verfrüht, aufgrund solcher bisher noch wenig sicherer Befunde endgültige Schlüsse zu ziehen bezüglich der empirischen Angemessenheit der verschiedenen theoretischen Vorhersagen, aber dennoch scheint beim gegebenen Wissensstand eher die Auffassung gestützt zu werden, die keine substantiellen Einflüsse der Bildung auf die Einkommensverteilung erwartet.

Berufe oder die Berufsstruktur ${ }^{10}$ - obwohl im Titel dieses Aufsatzes erwähnt - bleiben in der expliziten Argumentation der Theorien mehr oder weniger ausgespart. Auch die Theorie, die von der Struktur und Entwicklung der Arbeitsteilung her argumentiert, mißt der möglichen Macht von Berufsgruppen bzw. ihren Vereinigungen kaum Bedeutung bei. Aber Berufsverbände mögen sehr wohl die Verteilung der Löhne und Gehälter, wie sie aus dem reinen Modell der organisationellen Arbeitsteilung abgeleitet wird, beeinflussen. Verschiedene Berufsgruppen dürften Richtlinien für die bildungsmäßigen Voraussetzungen ihrer Mitglieder aufstellen (die auch in etlichen Fällen vom Staat überwacht oder sogar geregelt werden). Und die Berufsverbände verhandeln andererseits kollektiv mit den Arbeitgebern über Löhne und Gehälter. Deshalb dürften solche Berufsgruppen eine hier unberücksichtigt gebliebene Rolle bei der Verknüpfung von Bildung und Einkommen spielen.

10 Die Begriffe „Berufsstruktur“ und „Struktur der Arbeitsplätze" sind zu unterscheiden. Ersterer Begriff bezieht sich auf eine weitgefaßte Vorstellung von einem gesellschaftlichen Qualifizierungssystem, für das die allgemeine und berufsbezogene Bildung und die Professionalisierung bedeutsam sind, letzterer auf das Beschäftigungssystem, das durch die Logik der Unternehmensentwicklung gesteuert wird.
Die diesem Aufsatz zugrundeliegende Frage betrifft auch zentral die Instrumentalität der Bildung. Zwei sehr verschiedene Sichtweisen sind dazu in den Theorien vorhanden. Eine Theorie argumentiert auf der Grundlage einer ,inhaltlichen Instrumentalität" im Rahmen der Produktionsfunktion. Die andere Theorie geht von einer ,sozial definierten Instrumentalität“ im Rahmen der Legitimierung der Sozialstruktur aus. Die beiden Versionen einer Auswahltheorie der Bildung verbinden Vorstellungen beider Art.

Abgesehen von einer, inhaltlichen Instrumentalität" im Rahmen der Produktionsfunktion oder einer ,sozial definierten“" im LegitimierungsprozeB repräsentiert die Bildung auch einen gesellschaftlichen Wert an sich für die Mitglieder der Gesellschaft, was auch eine Entsprechung im Wertgefüge der Bildungsinstitutionen hat. Zudem mag Bildung auch eine Bewußtseinserweiterung bewirken und Quelle oder Stütze von Ideologien sein. Dies sind Fragestellungen der Soziologie, die in diesem Aufsatz ausgeklammert blieben ${ }^{11}$.

\section{Literatur}

Atkinson, A.B., 1975: The Economics of Inequality. Oxford: Clarendon Press.

Becker, Gary S., 1964: Human Capital. A Theoretical and Empirical Analysis With Special References to Education. New York: National Bureau of Economic Research. Second edition 1975.

Becker, Gary S./Chiswick, Barry R., 1966: Education and the Distribution of Earnings. American Economic Review 56: 358-369.

Blau, Peter M./Duncan, Otis, D., 1967: The American Occupational Structure. New York: Wiley.

Bornschier, Volker, 1976: Wachstum, Konzentration und Multinationalisierung von Industrieunternehmen. Reihe Soziologie in der Schweiz. Frauenfeld und Stuttgart: Huber.

Bornschier, Volker, 1977: Arbeitsteilung und soziale Ungleichheit. Kölner Zeitschrift für Soziologie und Sozialpsy chologie 29: 438-460.

Bornschier, Volker, 1981: Arbeitsteilung, strukturelle Mobilität und Klassenbildung. Eine theoretische Perspektive für die Mobilitätsforschung. Zeitschrift für Soziologie 10: 117-132.

11 Ausgeklammert bleiben mußten wegen der raschen Drucklegung auch Überlegungen zu den unterschiedlichen gesellschafts- und bildungspolitischen Konsequenzen der Theorien. 
Bornschier, Volker/Heintz, Peter, 1977: Statusinkonsistenz und Schichtung. Eine Erweiterung der Statusinkonsistenztheorie. Zeitschrift für Soziologie 6: 29-48.

Griliches, Zvi, 1977: Estimating the Returns to Schoolling: Some Econometric Problems. Econometrica 45: $1-22$.

Headey, Bruce/O'Loughlin, Tim, 1978: Transgenerational, ,Structured' Inequality: Social Fact or Fiction? British Journal of Sociology 29: 111-120.

Heintz, Peter, 1972a: Theory of Societal Systems. S. 127-139 in: Peter Heintz (Hrsg.): A Macrosociological Theory of Societal Systems. Band 1. Bern/ Stuttgart/Wien: Huber.

Heintz, Peter, 1972b: Structural and Anomic Tensions. S. 140-148 in: P. Heintz (Hrsg.): A Macrosocioloyical Theory of Societal Systems. Band 1. Bern/ Stut tgart/Wien: Huber.

Heintz, Peter, 1982: Ungleiche Verteilung. Macht und Legitimität. Möglichkeiten und Grenzen der strukturtheoretischen Analyse. Reihe Soziologie in der Schweiz. Diessenhofen: Rüegger.

Lord, George F./Falk, William W., 1980: An Exploratory Analysis of Individualist Versus Structuralist Explanations of Income. Social Forces 59: 376391.

Lydall, Harold F., 1968: The Structure of Earnings. Oxford: Clarendon Press.

Lydall, Harold F., 1976: Theories of the Distribution of Earnings. S. 15-46 in: A.B. Atkinson (Hrsg.): The Personal Distribution of Incomes. London: Allen and Unwin.

Mincer, Jacob, 1974: Schooling, Experience, and Earnings. New York: National Bureau of Economic Research.

Mincer, Jacob, 1976: Progress in Human Capital Analyses of the Distribution of Earnings. S. 136-192 in: A.B. Atkinson (Hrsg.): The Personal Distribution of Incomes. London: Allen and Unwin.

Müller, Walter, 1975: Familie - Schule - Beruf. Analysen zur sozialen Mobilität und Statuszuweisung in der BRD. Opladen: Westdeutscher Verlag.

Psacharopoulos, George, 1977: Family Background, Education and Achievement: A Path Model of Earnings Determinants in the U.K. and Some Alternatives. British Journal of Sociology 28: 321 335.

Reder, Melvin W., 1969: A Partial Survey of the Theory of Income Size Distribution. S. 203-251 in: Lee Soltow (Hrsg.): Six Papers on the Size Distribution of Income. New York und London: Columbia University Press.

Robinson, Robert V./Kelley, Jonathan, 1979: Class as Conceived by Marx and Dahrendorf: Effects on Income Inequality and Politics in the United States and Great Britain. American Sociological Review 44: 38-58.

Sahota, Gian Singh, 1978: Theories of Personal Income Distribution: A Survey. Journal of Economic Literatur 16: 1-55.

Thurow, Lester C., 1975: Generating Inequality: Mechanics of Distribution in the U.S. Economy. New York: Basic Books.

Thurow, Lester C., 1978: Die Arbeitskräfteschlange und das Modell des Arbeitsplatzwettbewerbs. S. 117-137 in: Werner Sengenberger (Hrsg.): Der gespaltene Arbeitsmarkt. Probleme der Arbeitsmarktsegmentation. Frankfurt und New York: Campus.

Wilson, Kenneth L., 1978: Toward an Improved Explanation of Income Attainment: Recalibrating Education and Occupation. American Journal of Sociology 84: 684-697. 